\title{
AUTOMATED EVALUATION OF CONTACT ANGLES IN A THREE- PHASE SYSTEM OF SELECTIVE AGGLOMERATION IN LIQUIDS
}

\author{
JULiA SCHREIER $^{1 *}$, ORKun FURAT ${ }^{\bowtie, 2^{*}}$, MURAT CANKAYA ${ }^{2}$, VOLKER SCHMIDT $^{2}$, \\ ULRICH BRÖCKEL ${ }^{1}$ \\ ${ }^{1}$ University of Applied Sciences Trier, Inst. f. Micro-Process-Engineering and Particle Technology (IMiP), \\ Umwelt-Campus Birkenfeld, P.O. Box 1380, D-55761 Birkenfeld, Germany; ${ }^{2}$ Ulm University, Institute of \\ Stochastics, D-89069 Ulm, Germany \\ *These authors have equally contributed to the work. \\ e-mail: orkun.furat@uni-ulm.de,j.schreier@umwelt-campus.de,murat.cankaya@uni-ulm.de, \\ volker.schmidt@uni-ulm.de,u.broeckel@umwelt-campus.de \\ (Received April 24, 2020; revised August 13, 2020; accepted August 24, 2020)
}

\begin{abstract}
This study aims to an automated evaluation of contact angles in a three-phase system of selective agglomeration in liquids. Wetting properties, quantified by contact angles, are essential in many industries and their processes. Selective agglomeration as a three-phase system consists of a suspension liquid, a heterogeneous solid phase and an immiscible binding liquid. It offers the chance of establishing more efficient separation processes because of the shape-dependent wetting properties of fine particles (size $\leq 10 \mu \mathrm{m}$ ). In the present paper, an experimental setup for contact angle measurements of fine particles based on the Sessile Drop Method is described. Moreover, a new algorithm is discussed, which can be used to automatically compute contact angles from image data captured by a high-speed camera. The algorithm uses a marker-based watershed transform to segment the image data into regions representing the droplet, the carrier plate coated by fine particles, and the background. The main idea is a parametric modelling approach for the time-dependent droplet's contour by an ellipse. The results show that the development of the dynamic contact angles towards a static contact angle can be efficiently determined based on this novel technique. These findings are useful for a detailed discrimination of wetting properties of spherical and irregularly shaped particles as well as their wetting kinetics. Also, a better understanding of selective agglomeration processes will be promoted by this user-friendly method.
\end{abstract}

Keywords: Automated detection, contact angle, image data, parametric contour modelling, selective agglomeration, three-phase system.

\section{INTRODUCTION}

Wetting properties of surfaces and methods for their measurement are essential in many industries. Examples can be found in coating processes, e.g., in the food (Zhang et al., 2019) or pharmaceutical industry (Karde and Ghoroi, 2014) in order to extend shelf life, enhance handling properties or reduce water vapor permeability (Kokoszka et al., 2010). Wetting properties play also an important role when agrochemicals are sprayed on leaves (Taylor, 2011) or when raw materials are processed by froth flotation (Albijanic et al., 2010; Debacher and Ottewill, 1991; Gharabaghi and Aghazadeh, 2014; Kowalczuk and Zawala, 2016; Newcombe and Ralston, 1994; Nguyen et al., 1997; Ralston et al., 2002). Moreover, the efficiency of granulation processes, expressly wet granulation processes or the spherical agglomeration (Farnand et al., 1961; Sirianni et al., 1969), depend on the wetting properties of solids characterized by the so-called contact angle. The spherical agglomeration process offers the possibility of a selective agglomeration of one specific solid component out of a heterogeneous suspension (Aktaş, 2002; Bensakhria et al., 2001). Akcil et al. (2009) showed that a selective agglomeration of gold out of a suspension of mineral matter, coal and gold particles results in a gold recovery of up to $95.5 \%$. Typically, primary particle sizes for spherical agglomeration processes range from $0.1 \mu \mathrm{m}$ up to $425 \mu \mathrm{m}$ (Dawei et al., 1986; Drzymala et al., 1991; Gürses et al., 1996; House and Veal, 1989; Kelsall and Pitt, 1987; Laskowski and Yu, 2000; Petela et al., 1995; Sadowski, 1995; Sirianni et al., 1969; Slaghuis and Ferreira, 1987; Wahl and Baker, 1971). 
Nowadays selective agglomeration is facing an increasing interest because it offers the chance of establishing more efficient separation processes. In the growing field of battery recycling or recycling of displays fine particles (size $\leq 10 \mu \mathrm{m}$ ) of valuable components can be selectively agglomerated depending on their wetting properties. The basic mechanism in these three-phase systems (suspension liquid / solid matter / binding liquid) is the interaction between dispersed droplets and suspended particles. According to their contact angles and the wetting kinetics (Bröckel, 1991) the interaction results in adherence or in separation from each other. The binding liquid must meet two main requirements. First an immiscibility gap towards the suspension liquid is a need. Second the suspended particles should preferably become wetted by the binding liquid.

Typically, the spherical agglomeration process is carried out in an intensively mixed system, e.g., a stirred vessel. After addition of a finely dispersed binding liquid to the suspension the agglomeration process starts (Madec et al., 2002). During intensive stirring particles collide with binding liquid droplets. If wetting properties are poor, particles and droplets separate from each other after collision. If the collision is successful and wetting occurs, particles stick together due to capillary forces and finally form agglomerates. The resulting agglomerates can be separated by sieving from the remaining suspension and used for further processing.

In a heterogeneous suspension containing two or more different solid components selectivity is determined by the contact angles between the individual solid components and the binding liquid. Components with a smaller wetting angle are preferably wetted and thereof agglomerated. Surface morphology (Morrow, 1970), addition of surface-active substances (Dawei et al., 1987) or change of $\mathrm{pH}$-value (House and Veal, 1989) of the suspension are factors that affect and modify the wettability of particles. In general, the contact angle is used to quantify the wetting of a substrate by a liquid in a surrounding continuous third phase (liquid or gas). According to Young's equation which is given by

$$
\cos \delta=\left(\gamma_{s g}-\gamma_{s l}\right) / \gamma_{l g},
$$

the contact angle $\delta$ depends on the involved surface tensions $\gamma_{\mathrm{ij}}$ with the subscripts $s$ (solid), $g$ (gas or a second liquid) and $l$ (liquid droplet). These surface tensions interact at the three-phase contact line, which is detected at two points in the front view (see Fig. 1). Then, by means of Eq. (1), the contact angle $\delta$ is defined as the angle between the tangents on the solid surface and the contour of the droplet starting at the three-phase contact points.

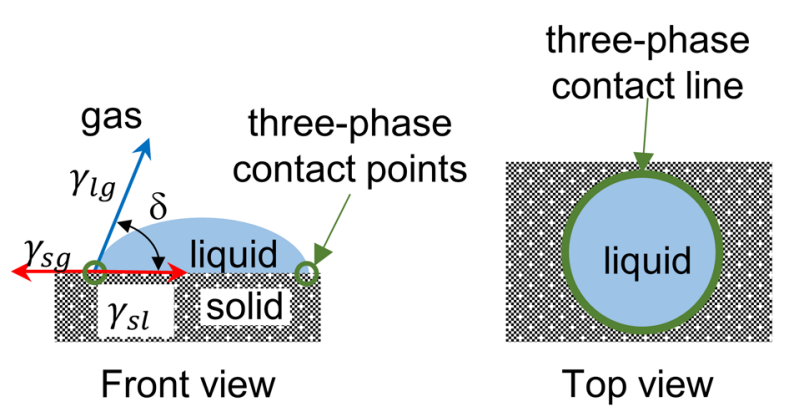

Fig. 1. Wetting of a solid surface by a liquid

A common method for determining contact angles on flat surfaces is the Sessile Drop Method (Alghunaim et al., 2016; Buckton and Newton, 1986; Kossen and Heertjes, 1965; Nowak et al., 2013; Santini et al., 2013) where a droplet is placed on a solid surface (Fig. 1). The contact angle in front view direction is then measured by means of user-driven image processing techniques (Lazghab et al., 2005), which is a time-consuming procedure being afflicted with subjective measurement errors. It is based on the following physical phenomena. When a droplet is placed on a solid surface, the wetting process starts and the contact line is moving. During this movement, molecules of the droplet displace molecules from the surrounding fluid already adhered to the solid surface. The expansion speed of the three-phase contact line can be determined, when the wetting process itself is recorded with a high-speed camera. Finally, after equilibrium of surface forces is reached expansion of the three-phase contact line terminates and the static contact angle can be measured.

Furthermore, in literature numerous attempts to measure contact angles of powders are described (Alghunaim et al., 2016; Bröckel and Löffler, 1991; Buckton and Newton, 1986; Link and Schlünder, 1996; Saulick et al., 2017). One of these approaches is based on compacting powdery particles into a tablet and measuring the contact angle on the surface of this tablet (Buckton and Newton, 1986). A major disadvantage of this technique is the dependence of the contact angle on the applied pressure during tableting. This compaction pressure causes breakage of primary particles and may also alter the surface morphology of these particles.

On the other hand, a quite simple method is fixing particles with glue (Bröckel and Löffler, 1991) or double-sided adhesive tape (Saulick et al., 2017) on a carrier plate (e.g. objective slide). The advantage of this method is that surface morphology of the particles is not affected by preparation. Moreover, using double-sided adhesive tapes eliminates the possibility of changing the wetting properties caused by a flowable glue spreading over particles during preparation. The insolubility of compo- 
nents of the adhesive tape in suspension and binding liquid was proven in advance. Thus, no effect of the adhesive tape on the wetting properties is to be expected. For determining contact angles of a droplet of binding liquid in contact with a layer of particles (size $<10 \mu \mathrm{m}) \mathrm{sub}-$ mersed in suspension liquid, a device based on the Sessile Drop Method described above was developed. The movement of the three-phase contact line and the change of droplet contour from the very beginning until steady state has been recorded with a high-speed camera, see Section Experimental for details.

The aim of the present paper is to introduce a new algorithm to automatically determine the contact angle via image processing in order to efficiently study the influence of particle morphology on the contact angle during wetting, see Section Automated Angle Detection. The algorithm uses a marker-based watershed transform to segment image data captured by a high-speed camera into regions representing the droplet, the carrier plate coated by fine particles, and the background (Soille, 2013; Spettl et al., 2015). The main idea is a parametric modelling approach, where a line and an ellipse are fitted to the pixels associated with the front view of the carrier's surface and the droplet surface, respectively. The contact angles are then computed analytically using the parametric representation of the line and ellipse. The results show that the development of the dynamic contact angles towards a static contact angle can be efficiently determined based on this novel technique.

\section{MATERIALS AND METHODS}

\section{EXPERIMENTAL PROCEDURES}

To determine the influence of the surface morphology of particles on the wetting properties two different types of hydrophobic particles, namely natural platelet and spherical graphite particles (see Table 1), were considered. Water was acting as suspension liquid, paraffin oil as a binding liquid.

As already mentioned in Section Introduction, a measurement device based on the Sessile Drop Method was developed, which consists of a glass plate as carrier plate where one side of the glass plate is covered with double-sided adhesive tape. The adhesive tape was coated with particles without applying any additional pressure, see Fig. 3. Particles not adhering on the adhesive tape were removed by a soft air stream. This procedure results in a densely packed monolayer of particles on the carrier plate. Next the carrier plate is perpendicularly immersed in a water-filled basin at room temperature. Under the water the carrier plate is rotated horizon-

Table 1. Material characteristics of two different graphite types where $x_{10}, x_{50}$ and $x_{90}$ denote the 10-, 50- and 90quantiles of the size distribution, respectively.

\begin{tabular}{|c|c|c|c|c|c|c|}
\hline Shape & Company & Type & $\begin{array}{c}x_{10} \\
{[\mu \mathrm{m}]}\end{array}$ & $\begin{array}{c}x_{50} \\
{[\mu \mathrm{m}]}\end{array}$ & $\begin{array}{c}x_{90} \\
{[\mu \mathrm{m}]}\end{array}$ & $\begin{array}{c}\text { Purity } \\
{[\%]}\end{array}$ \\
\hline Platelet & AMG Graphite & UF2 99.9 & 2.0 & 4.4 & 7.9 & 99.98 \\
\hline Spherical & ProGraphiteShop & $1110-1$ & 6.3 & 11.2 & 19.0 & 99.95 \\
\hline
\end{tabular}
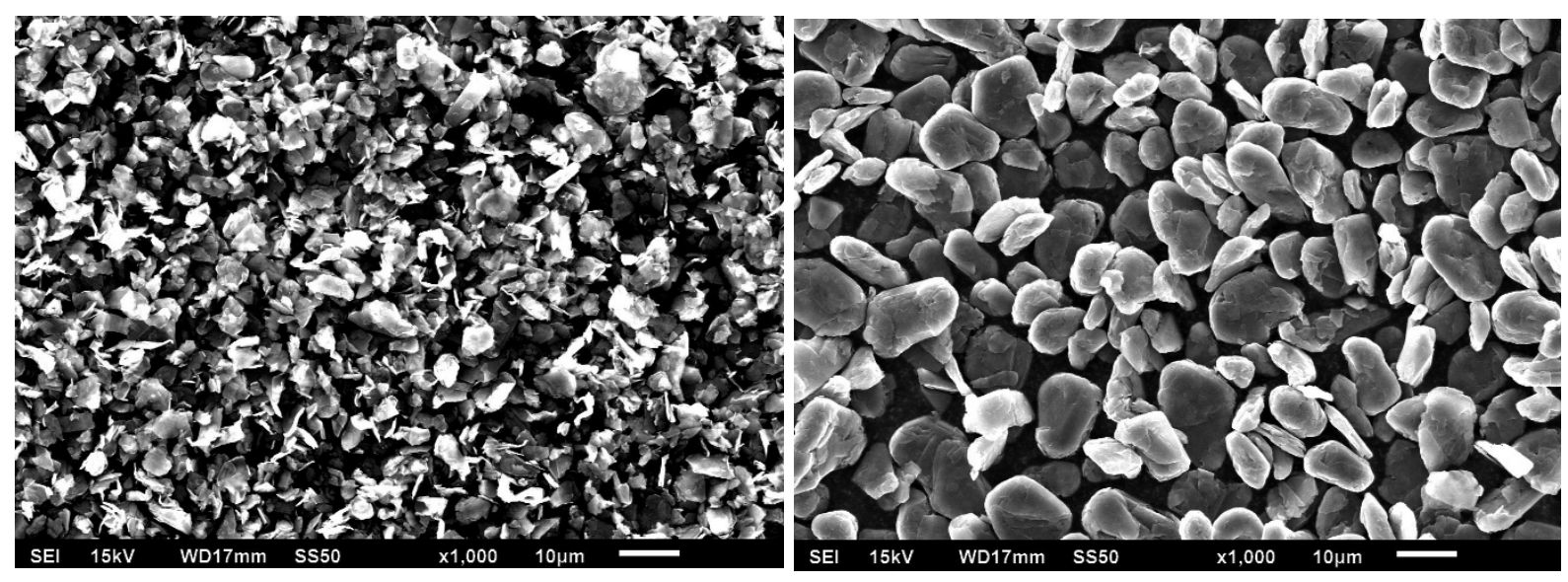

Fig. 2. SEM images of graphite particles; left: platelet graphite, right: spherical graphite. 


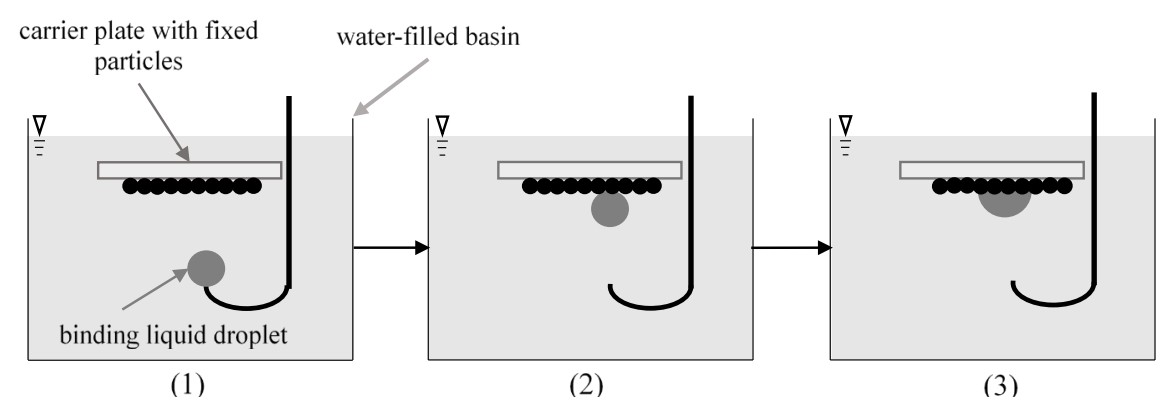

Fig. 3. Measurement device for determining contact angles during collisions; (1) creation of droplet from binding liquid, (2) contact of droplet with particles, (3) wetting of the particles by the droplet.

tally, so that the particle coated side is upside down. During immersion into water gas bubbles may adhere to the coated surface. In order to reduce the negative influence of gas bubbles water was degassed for 20 minutes with a water jet pump prior to measurement. Thus, eventually adhering gas bubbles will dissolve in the degassed water.

Through a needle, which is fixed on a micro syringe a droplet of highly liquid paraffin oil (Merck KGaA, $\left.\rho=0.85 \mathrm{~g} / \mathrm{cm}^{3}\right)$ is inflated until the buoyancy force is large enough so that the droplet detaches from the needle. The droplet moves upwards and attaches on the particle monolayer (see Fig. 3). After this collision the contact area between droplet and particles increases until equilibrium of the interacting surface forces (see Fig. 1, left). A high-speed camera (Keyence, type VW-600M, $250 \mathrm{fps}$ ) records this process to determine when the temporal evolvement from a dynamic contact angle to the final static contact angle is finished.

For each particle morphology four carrier plates coated with particles were examined. Common open source image processing programs like the ImageJ plugin "contact angle" (Brugnara, 2010; Schneider et al., 2012) require a manual selection of initial points on the contour of the droplet and a decision on the three-phase contact points by the user. This is a time-consuming procedure and the results are affected by user decisions. In our forthcoming studies a large number of contact angle measurements is planned. Thus, the development of a user-independent and automated contact angle detection procedure was favorable.

\section{AUTOMATED ANGLE DETECTION}

In this section, we describe an algorithm, which can be used to automatically compute contact angles from front-view image data captured by a high-speed camera, see Fig. 8. Note that for varying experimental setups such an automated detection scheme may require some readjustments. For instance, this may be the case when using a different camera or when changing the position of the camera/light source. The algorithm consists of the following steps:

i. Detection of the region of interest and cropping of the image data.

ii. Extraction of pixels associated with the carrier's surface, followed by fitting a line to these pixels.

iii. Detection of pixels which lie on the interface between the droplet and the background. Then, an ellipse is fitted to the extracted pixels.

iv. Computation of intersection points between the fitted line and ellipse. By determining the tangents of the ellipse at the intersection points, the contact angles can be obtained by computing the angles between the fitted line and the tangents.

From now on, we denote the series of $n$ grayscale images captured in a single experiment by $I_{1}, \ldots, I_{n}$. Furthermore, an image $I$ of size $m \times k$ is considered to be a function $I: \mathbb{Z}^{2} \rightarrow \mathbb{R}$ with $I(x, y)=0$ for each $(x, y) \notin$ $\{1, \ldots, m\} \times\{1, \ldots, k\}$.

\section{Detection of the region of interest}

In order to make the subsequent steps easier, it is useful to crop the images $I_{t}$ obtained by the high-speed camera, such that, for each time-step $t=1, \ldots, n$, we obtain a cutout $C_{t}$ of $I_{t}$ which only depicts the region of interest relevant for computing contact angles.

Therefore, in a first step, from the raw data a rough cutout $R_{t}$ of $I_{t}$ is taken, where the position and size of the window for cropping the image $I_{t}$ was predefined in such a way that the contact area is included in the cutout, see Fig. 4 (left). Then, we compute the gradient magnitude image $G_{t}$ of $R_{t}$ which is given by

$$
G_{t}=\sqrt{\left(R_{t} * k_{x}\right)^{2}+\left(R_{t} * k_{y}\right)^{2}},
$$



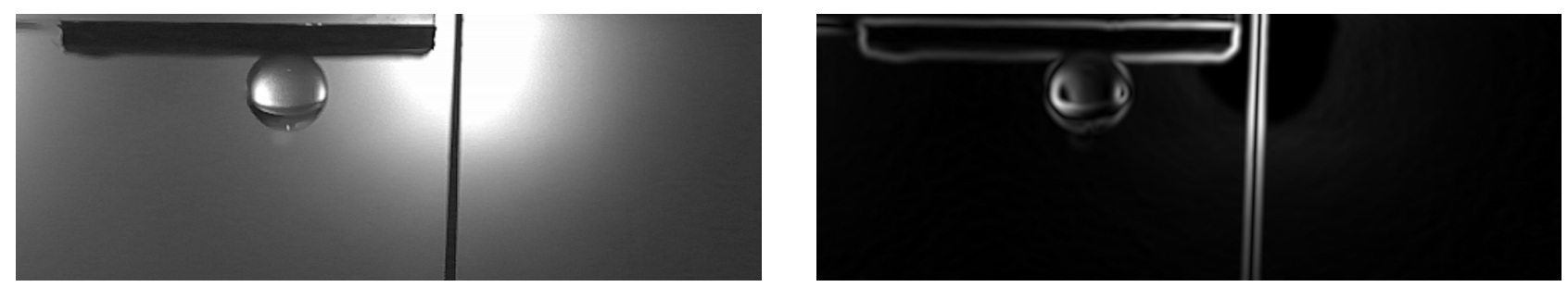

Fig. 4. left: "Rough” cutout of the region of interest. right: Corresponding gradient magnitude image.

where * denotes the discrete convolution operation and $k_{x}$ and $k_{y}$ are Sobel operators in $x$ - and $y$-direction, respectively (Soille, 2013), see Fig. 4 (right). Note that the discrete convolution of $R_{t}$ with $k_{x}$ and $k_{y}$ approximates the partial derivatives of a space-continuous analogon of $R_{t}$.

From the gradient magnitude image $G_{t}$ it is relatively easy to characterize landmarks, like the horizontal surface of the carrier plate or the vertical needle, such that a more precise cutout of the contact area can be taken. More precisely, we compute projections $G_{t}^{(x)}$ and $G_{t}^{(y)}$ of $G_{t}$ in $x$-and $y$-direction, respectively, which are given by

$$
G_{t}^{(x)}(y)=\sum_{x=-\infty}^{\infty} G_{t}(x, y)
$$

and

$$
G_{t}^{(y)}(x)=\sum_{y=-\infty}^{\infty} G_{t}(x, y)
$$

Smoothed versions of the projections $G_{t}^{(y)}$ and $G_{t}^{(x)}$ of the gradient magnitude image depicted in Fig. 4 (right) are visualized in Fig. 5 (left and middle). From the local maxima of $G_{t}^{(y)}$ it is possible to approximately derive the $x$-coordinates of the vertical needle, and of the most left and right points belonging to the droplet. On the other hand, the projection $G_{t}^{(x)}$ gives us information about the $y$-coordinate of the horizontal surfaces belonging to the carrier plate. With this information about certain image landmarks and with some assump- tions on the droplet size, we are able to derive a relatively small rectangular bounding box $W_{t} \subset \mathbb{Z}^{2}$ for the droplet at each time-step $t=1, \ldots, n$. Since the droplet's movement during the measurement is rather limited, we derive a common bounding box $W$ for the entire sequence of images, by computing median values of the coordinates of diagonally opposing corner points of the boxes $W_{t}$. Finally, we obtain the image $C_{t}$, which depicts the region of interest, by cropping $I_{t}$ with the bounding box $W$, see Fig. 5 (right).

Line-fitting to the front view of carrier plate's surface

In this section, we describe further image processing steps for determining a line representing the carrier plate's surface. In order to do so, we enhance the cropped images $C_{t}$ by performing contrast-limited adaptive histogram equalization (Zuiderveld, 1994) followed by denoising with the non-local means algorithm $(\mathrm{Bu}-$ ades et al., 2005), see Fig. 6 (i and ii). For the sake of simplicity, we denote these preprocessed images again by $C_{t}$. Since the position of the carrier plate and the highspeed camera is fixed during the experiment, we assume that a single line $g$ can represent the carrier plate's surface for each time-step $t=1, \ldots, n$. Therefore, we use a time-averaged image $\bar{C}$, which is given by

$$
\bar{C}=\frac{1}{m} \sum_{t=1}^{m} C_{t}
$$

for some $m \leq n$. This enhances the contrast even further, see Fig. 6 (iii). Then, by means of a transformation
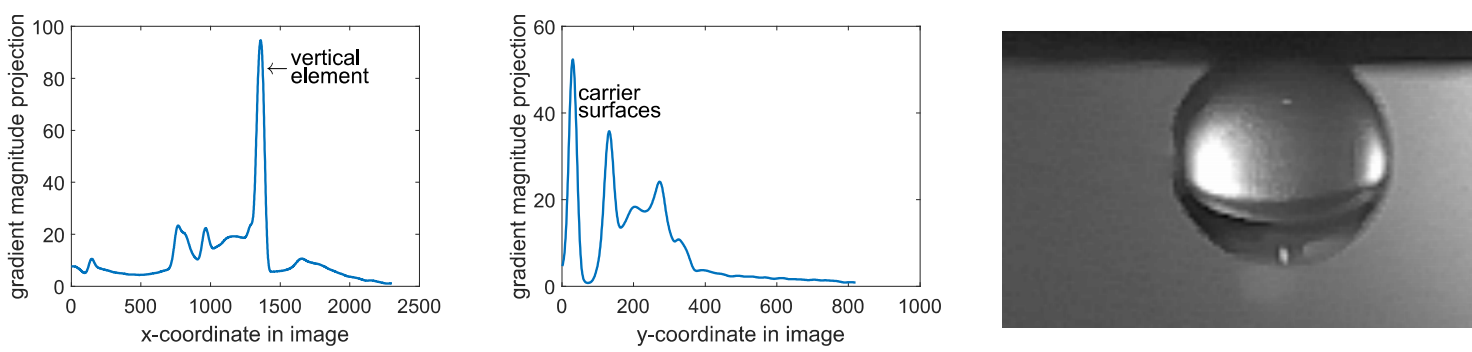

Fig. 5. Smoothed projections of the gradient magnitude in y-direction (left) and x-direction (middle). Right: Final cutout $C_{t}$ taken from $R_{t}$ depicted in Fig. 4 (left). 
similarly to Eq. (2) the gradient magnitude image of $\bar{C}$ is computed from which a binary image $\mathrm{E}$ depicting the edges of $\bar{C}$ is extracted using the Canny algorithm (Canny, 1986), see Fig. 6 (iv).

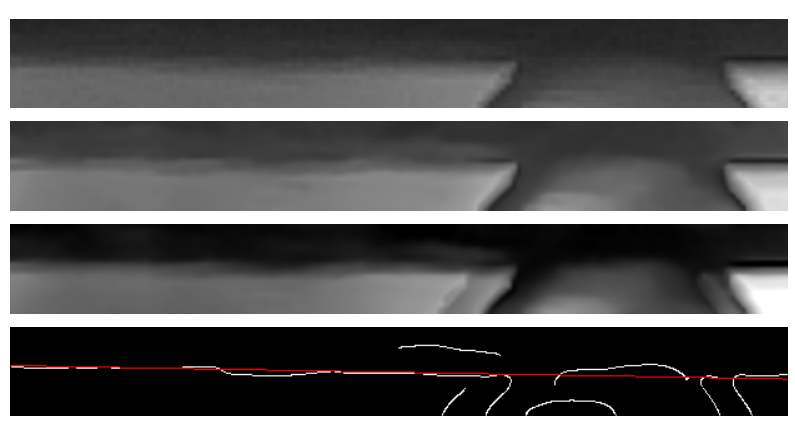

Fig. 6. From top to bottom: (i) Cutout of the raw image data used for the detection of the carriers surface. (ii) Preprocessed image. (iii) Time-averaged gradient magnitude image. (iv) Binarized edge image E computed by means of the Canny algorithm and the detected line (red).

Finally, the Hough transform (Gonzalez et al., 2004 ) is computed from the edge image $E$ in order to determine a line $g$ given by

$$
g(x)=\alpha x+\beta
$$

which represents the surface of the carrier plate for some $\alpha$ and $\beta$, see Fig. 6 (iv, red line).

Extraction and parametric modelling of the droplet's contour

Now we describe the procedure for extracting the droplet's contour from the cropped preprocessed image data, see Fig. 7 (i). More precisely, we detect the left and right contour of the droplet in two independent steps. We only describe the procedure for determining the left contour, since the detection of the droplet's right contour is performed analogously. Therefore, in a first step, the left contour is enhanced by computing an edge image $E_{t}$ which is given by

$$
E_{t}=\sum_{k=1}^{5}\left|C_{t} * g^{(k)}\right|,
$$

where $g^{(1)}, \ldots, g^{(5)}$ are convolution kernels used in the Kirsch operator, see (Kirsch, 1971).

Each of these kernels is chosen such that the convolution operation leads to an enhancement of specifically oriented fragments of the edges, see Fig. 7 (ii). Then, a marker-based watershed transform is computed (Soille, 2013; Spettl et al., 2015) to segment the image into the background left of the droplet and the remaining image domain. For this segmentation procedure, we define two disconnected regions as marks, from which a region growth is performed to obtain the segmentation. The

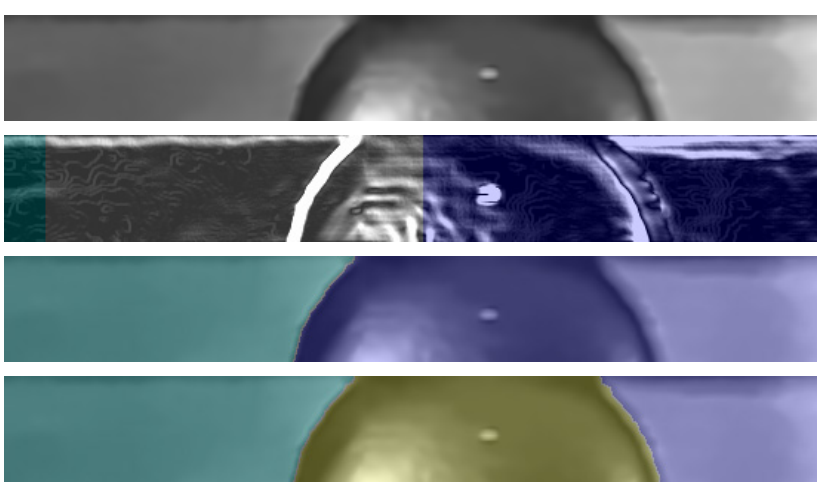

Fig. 7. From top to bottom: (i) Cutout of the preprocessed image obtained by histogram equalization and non-local means denoising. (ii) Image with enhanced left droplet surface obtained by utilizing convolution kernels in the Kirsch operator and markers used in the marker-based watershed algorithm (green, purple). (iii) Segmentation for determining the left contour of the droplet obtained by applying the watershed algorithm on the image and markers. (iv) Final segmentation of the droplet.

marker, which corresponds to the background, only covers the background on the left-hand side of the image. The second marker covers the area between the droplet centre and the right edge of the image, see Fig. 7 (ii). The segmentation result obtained after the regiongrowth procedure is depicted in Fig. 7 (iii). The left edge of the droplet is then described by the pixels located at the interface between the two regions. Analogously, we determine the right edge of the droplet-leading to the final segmentation of the droplet, see Fig. 7 (iv). Thus, from each segmented image denoted by $S_{t}$, we can extract a set of pixels $B_{t \text {,surface }} \subset \mathbb{Z}^{2}$ associated with the contour of the droplet's surface. In order to describe the contour parametrically, we fit an ellipse to the pixels in $B_{t, \text { surface }}$ Therefore, we denote the set of all ellipse parameterizations by $\Theta \subset \mathbb{R}^{5}$, where each five-dimensional vector $\theta=\left(x_{c}, y_{c}, a, b, \varrho\right) \in \Theta$ describes an ellipse $H_{\theta}$ by its centre $\left(x_{c}, y_{c}\right)$, the lengths of its halfaxes $a, b$ and its rotation angle $\varrho$. The optimal ellipse $H_{\theta_{t}}$, which has the smallest discrepancy to the set of droplet's boundary pixels $B_{t, \text { surface }}$, is obtained by minimizing the discrepancy

$$
\theta_{t}=\operatorname{argmin}_{\theta \in \Theta} \sum_{z \in B_{t, \text { surface }}}\left\|z-P_{\theta}(z)\right\|^{2},
$$

where $\|\cdot\|$ denotes the Euclidean norm and $P_{\theta}(z)$ is the boundary point of the ellipse $H_{\theta}$ which is closest to $z \in \mathbb{R}^{2}$. Note that, in practice, the search for an optimal parameterization $\theta_{t}$ by means of Eq. (8) is only performed on a certain subset $\Theta^{\prime} \subset \Theta$ of all potentially possible ellipse parameterizations. More precisely, we constrain the parameter space $\Theta$, such that only reasonably sized and shaped ellipses $H_{\theta}$ are considered and whose 
centers are sufficiently close to the center of the image $E_{t}$.

\section{Computation of contact angles}

After describing the front view of the carrier's surface by the line $g$ given in Eq. (6) and the contour of the droplet's surface at time $t$ by the ellipse $H_{\theta_{t}}$, we can compute the contact angles using analytical formulas. Therefore, in a first step, we compute the intersection points between $g$ and $H_{\theta_{t}}$. Since the contact angles do not change, when rigidly transforming $g$ and $H_{\theta_{t}}$ in the same manner, we can easily rotate and shift the line and ellipse such that the latter's center is located at the origin of the coordinate system and that its half-axes are parallel to the coordinate system's axes. Thus, from here on, we only consider parameterizations of the form $\theta=$ $(0,0, a, b, 0)$. Then, by solving the quadratic equation

$$
\frac{x^{2}}{a^{2}}+\frac{(\alpha x+\beta)^{2}}{b^{2}}=1
$$

we obtain the $x$-coordinates $x_{\ell}$ and $x_{r}$ of the left and right intersection points, respectively.

The corresponding $y$-coordinates are given by $y_{\ell}=$ $g\left(x_{\ell}\right)$ and $y_{r}=g\left(x_{r}\right)$. The outer normal vector $n_{\ell}$ of the ellipse at the left intersection point $\left(x_{\ell}, y_{\ell}\right)$ is given by

$$
n_{\ell}=\frac{\left(\frac{x_{\ell}}{a^{2}}, \frac{y_{\ell}}{b^{2}}\right)}{\sqrt{\frac{x_{\ell}^{2}}{a^{4}}+\frac{y_{\ell}^{2}}{b^{4}}}}
$$

By rotating $n_{\ell}$ counterclockwise by $\frac{\pi}{2}$, we obtain the tangential direction $\tilde{n}_{\ell}$ of the ellipse at the intersection point $\left(x_{\ell}, y_{\ell}\right)$, see Fig. 8 .

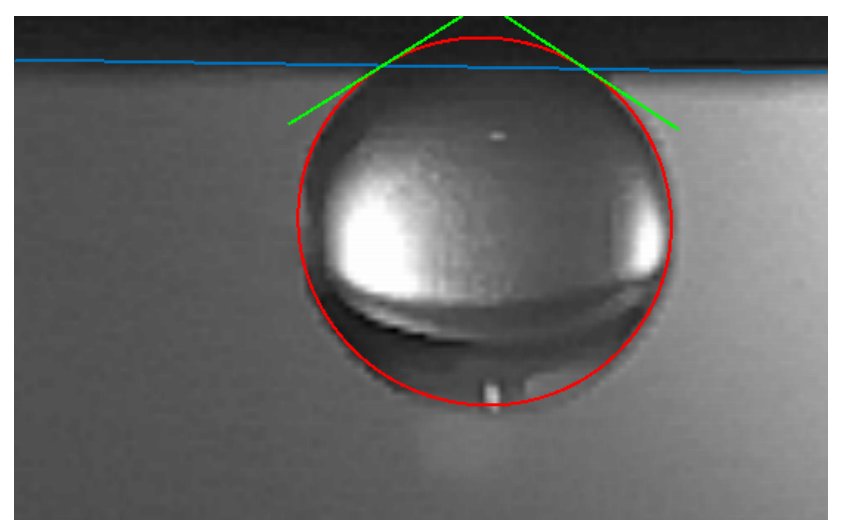

Fig. 8. Line (blue) and ellipse (red) describing the carrier's surface and the droplet's surface, respectively. The green lines indicate the tangents of the ellipse at the intersection points.

Finally, the left contact angle $\delta_{\ell}$ is given by the angle between the vector $\tilde{n}_{\ell}$ and the directional vector $n_{g}$ of $g$, i.e., we have

$$
\delta_{\ell}=\cos ^{-1}\left(\tilde{n}_{\ell} \cdot n_{g}\right)
$$

where $n_{g}=\frac{(1, \alpha)}{\sqrt{1+\alpha^{2}}}$. Analogously, the right angle $\delta_{r}$ is computed. In this manner, the left and right contact angles can be computed for a measurement based on a sequence of images by computing the contact angles of the ellipses $H_{\theta_{t}}$ and the line $g$ for each time-step $t=$ $1, \ldots, n$.

\section{RESULTS}

The development of the dynamic contact angles towards a static contact angle depends on the surface morphology of the carrier plate. In the experiments considered in the present paper, the surface morphology is closely related with the shape of the coating particles, which, consequently, influences the resulting contact angles. In Fig. 9 and Fig. 10 the contact angles obtained for carrier plates coated with particles of two different shapes over various time steps are plotted. Recall that for all experiments, the system consists of either spherically-shaped or platelet graphite particles, which are fixed on the carrier plate, with water as surrounding liquid and paraffin oil in form of liquid droplets, see Section Experimental. To reduce signal noise the computed contact angles depicted in Fig. 9 and 10 have been smoothed over time using a Gaussian filter.

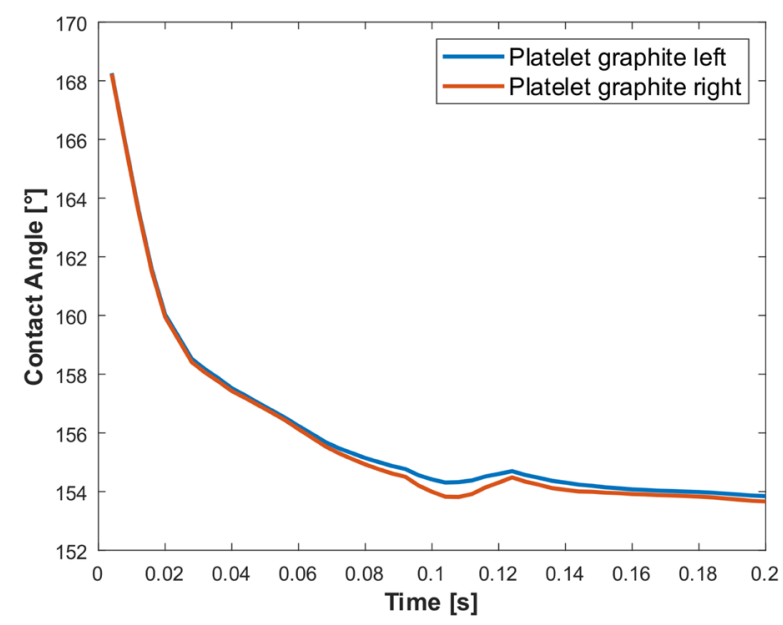

Fig. 9. Time-dependent contact angles of left- and righthand side of the droplet's contour for platelet graphite in the three-phase system graphite-water-paraffin-oil.

Note that in Fig. 9 the contact angles of the left- and right-hand side of the paraffin droplet show a sharp drop until $0.14 \mathrm{~s}$. After this, the contact angles reach the static regime, where the computed values decline very little over time. Furthermore, the differences between the contact angles of the left- and right-hand side are almost zero. This indicates a symmetry of the drop, which is 
why we focus on the average values of both sides in the following.

Comparing the two differently shaped particles, it is noticeable that the static contact angle regime of the spherically shaped particles is reached after $0.04 \mathrm{~s}$, but the static contact angle for the platelets after $0.14 \mathrm{~s}$ (see Fig. 10). This implies that the wetting kinetics of platelets is significantly slower compared to spherical particles.

Furthermore, the static contact angle of the platelet graphite $\left(153.9^{\circ} \pm 0.1^{\circ}\right)$ is $7.9^{\circ}$ larger than the static contact angle of the spherical graphite $\left(146.0^{\circ} \pm 0.5^{\circ}\right)$.

\section{DISCUSSION}

As shown in Section Results, spherical graphite particles have a faster wetting kinetic than platelet graphite particles. Consequently, the static contact angle of platelet graphite is $7.9^{\circ}$ larger than that of spherical graphite. This result is to be expected, because a more pronounced surface roughness, as in the case of the carrier plate prepared with platelet graphite, results in larger contact angles. Vice versa, a less pronounced surface roughness leads to smaller contact angles, as it can be observed in the experiments with spherical particles, see Fig. 10. The relationship between wettability and roughness of engineering surfaces was described by Kubiak et al. (2011). More precisely, they observed a reduction of contact angles for smoother surfaces, which is true for carrier plates coated with spherical particles.

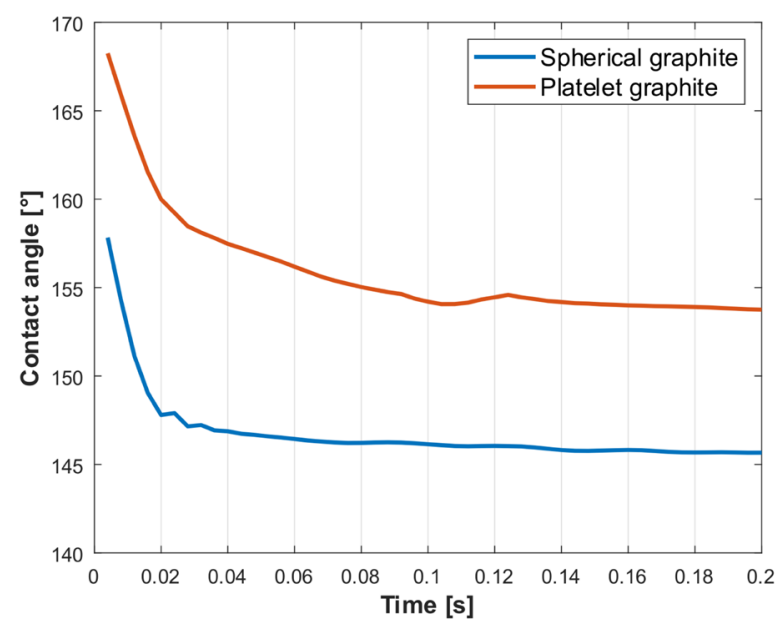

Fig. 10. Time-dependent contact angle of spherical and platelet graphite.

\section{CONCLUSION}

The main goal of this work was to develop a new algorithm for automated image processing, which evaluates grayscale images obtained during measurements with the Sessile Drop Method in order to efficiently determine the development of the dynamic contact angles towards a static contact angle. Furthermore, the developed method was applied to study the influence of particle morphology on the static contact angles in the system graphite-water-paraffin oil. We have shown that platelet particles lead to larger contact angles than spherical particles due to the increased surface roughness of carrier plates coated by platelet particles. Additionally, two different regimes of wetting kinetics have been identified. It turned out that in comparison to graphite platelets the wetting kinetics of spherical particles is approximately three times faster.

\section{ACKNOWLEDGEMENTS}

This work has been financially supported by the German Research Foundation (DFG), Priority Programme 2045

\section{REFERENCES}

Akcil A, Wu XQ, Aksay EK (2009). Coal-Gold Agglomeration: An Alternative Separation Process in Gold Recovery. Sep Purif Rev 38:173-201.

Aktaş Z (2002). Some factors affecting spherical oil agglomeration performance of coal fines. Int J Min Process 65:177-90.

Albijanic B, Ozdemir O, Nguyen AV, Bradshaw D (2010). A review of induction and attachment times of wetting thin films between air bubbles and particles and its relevance in the separation of particles by flotation. Adv Colloid Interface Sci 159:1-21.

Alghunaim A, Kirdponpattara S, Newby BZ (2016). Techniques for determining contact angle and wettability of powders. Powder Technol 287:201-15.

Bensakhria A, Sajet P, Antonini G, Auquier W (2001). Solid/solid separation by selective agglomeration with agglomerant recovery by thermal desorption. Chem Eng J 81:171-78.

Bröckel U (1991). Untersuchungen zur Benetzungskinetik zwischen Partikeln und Kollektoren bei der Umbenetzungsagglomeration in Flüssigkeiten. PhD Universität Karlsruhe (TH).

Bröckel U, Löffler F (1991). A technique for measuring contact angles at particles. Part Part Syst Charact $8: 215-21$.

Brugnara M (2010). Contact Angle Plugin, Italy. 
Buades A, Coll B, Morel JM (2005). A non-local algorithm for image denoising. In: 2005 IEEE Comput. Soc. Conf. Comput. Vis. Pattern Recognit. CVPR05, IEEE, San Diego, CA, USA: 60-5.

Buckton G, Newton JM (1986). Assessment of the wettability of powders by use of compressed powder discsPowder Technol 46:201-08.

Canny J (1986). A computational approach to edge detection, IEEE Transactions on pattern analysis and machine intelligence 20:679-98.

Dawei W, Kewu W, Jicun Q (1986). Hydrophobic agglomeration and spherical agglomeration of wolframite fines. Int J Miner Process 17:261-71.

Dawei W, Kewu W, Jicun Q (1987). The activation mechanisms of wolframite by $\mathrm{Ca} 2+$ and $\mathrm{Fe} 3+$ ions in hydrophobic agglomeration, using sodium oleate as collector. Int J Miner Process 20:35-44.

Debacher NA, Ottewill RH (1991). Kinetics of contact angle formation at the gas-liquid-solid interphase. Colloids Surf 52:149-61.

Drzymala J, Markuszewski R, Wheelock TD (1991). Oil Agglomeration of Sulfurizes Pyrite. Miner Eng 4:16172 .

Farnand JR, Smith HM, Puddington IE (1961). Spherical agglomeration of solids in liquid suspension. Can $\mathrm{J}$ Chem Eng 39:94-7.

Gharabaghi M, Aghazadeh S (2014). A review of the role of wetting and spreading phenomena on the flotation practice. Curr Opin Colloid Interface Sci 19:266-82.

Gonzalez RC, Woods RE, Eddins SL (2004). Digital Image Processing Using MATLAB, Pearson Education India.

Gürses A, Doymus K, Bayrakceken S (1996). Selective oil agglomeration of brown coal: a systematic investigation of the design and process variables in the conditioning step. Fuel 75:1175-80.

House CI, Veal CJ (1989). Selective recovery of chalcopyrite by spherical agglomeration. Miner Eng 2:171184.

Karde V, Ghoroi C (2014). Influence of surface modification on wettability and surface energy characteristics of pharmaceutical excipient powders. Int $\mathrm{J}$ Pharm 475:351-63.

Kelsall GH, Pitt JL (1987). Spherical agglomeration of fine wolframite $((\mathrm{Fe}, \mathrm{Mn}) \mathrm{WO} 4)$ mineral particles. Chem Eng Sci 42:679-88.

Kirsch RA (1971). Computer determination of the constituent structure of biological images. Computers and biomedical research 4:315-28.

Kokoszka S, Debeaufort F, Hambleton A, Lenart A, Voilley A (2010). Protein and glycerol contents affect physico-chemical properties of soy protein isolate- based edible films. Innov. Food Sci Emerg Technol 11:503-10.

Kossen NWF, Heertjes PM (1965). The determination of the contact angle for systems with a powder. Chem Eng Sci 20:593-99.

Kowalczuk PB, Zawala J (2016). A relationship between time of three-phase contact formation and flotation kinetics of naturally hydrophobic solids. Colloids Surf Physicochem Eng Asp 506:371-77.

Kubiak KJ, Wilson MCT, Mathia TG, Carval P (2011). Wettability versus roughness of engineering surfaces. Wear. 271:523-28.

Laskowski JS, Yu Z (2000). Oil agglomeration and its effect on beneficiation and filtration of low-rankroxidized coals. Int J Miner Process 58:237-52.

Lazghab M, Saleh K, Pezron I, Guigon P, Komunjer L (2005). Wettability assessment of finely divided solids. Powder Technol 157:79-91.

Link KC, Schlünder EU (1996). A new method for the characterisation of the wettability of powders. Chem Eng Technol 19:432-37.

Madec L, Muhr H, Plasari E (2002). Development of new methods to accelerate and improve the agglomeration of submicron particles by binding liquids. Powder Technol 128:236-41.

Morrow NR (1970). Physics and thermodynamics of capillary action in porous media, Ind Eng Chem 62:3256.

Newcombe G, Ralston J (1994). Bubble spreading kinetics and mineral flotation. Miner Eng 7:889-903.

Nguyen AV, Schulze HJ, Ralston J (1997). Elementary steps in particle-bubble attachment. Int J Miner Process 51:183-95.

Nowak E, Robbins P, Combes G, Stitt EH, Pacek AW (2013). Measurements of contact angle between fine, non-porous particles with varying hydrophobicity and water and non-polar liquids of different viscosities, Powder Technol 250:21-32.

Petela R, Ignasiak B, Pawlak W (1995). Selective agglomeration of coal: analysis of laboratory batch test results. Fuel 74:1200-10.

Ralston J, Dukhin SS, Mishchuk NA (2002). Wetting film stability and flotation kinetics. Adv Colloid Interface Sci95:145-236.

Sadowski Z (1995). Selective spherical agglomeration of fine salt-type mineral particles in aqueous solution. Colloids Surf 96:277-85.

Santini M, Guilizzoni M, Fest-Santini S (2013). X-ray computed microtomography for drop shape analysis and contact angle measurement. J Colloid Interface Sci 409:204-10. 
Saulick Y, Lourenço SDN, Baudet BA (2017). A semi-automated technique for repeatable and reproducible contact angle measurements in granular materials using the sessile drop method. Soil Sci Soc Am J 81:24149.

Schneider CA, Rasband WS, Eliceiri KW (2012). NIH Image to ImageJ: 25 years of image analysis. Nature methods 9:671-75.

Sirianni AF, Capes C., Puddington JE (1969). Recent experience with the spherical agglomeration process. Can J Chem Eng 47:166-70.

Slaghuis JH, Ferreira LC (1987). Selective spherical agglomeration of coal. Fuel. 66:1427-30.

Soille P (2013). Morphological Image Analysis: Principles and Applications. Springer Science \& Business Media.

Spettl A, Wimmer R, Werz T, Heinze M, Odenbach S, Krill CE, Schmidt V (2015). Stochastic 3D modeling of Ostwald ripening at ultra-high volume fractions of the coarsening phase. Model Simul Mater Sci Eng 23: 065001 .

Taylor P (2011). The wetting of leaf surfaces. Curr Opin Colloid Interface Sci 16:326-34.

Wahl EF, Baker CGJ (1971). The kinetics of titanium dioxide agglomeration in an agitated liquid suspension. Can J Chem Eng 49:742-46.

Zhang Y, Bi J, Wang S, Cao Q, Li Y, Zhou J, Zhu BW (2019). Functional food packaging for reducing residual liquid food: Thermo-resistant edible super-hydrophobic coating from coffee and beeswax. J Colloid Interface Sci 533:742-49.

Zuiderveld K (1994). Contrast limited adaptive histogram equalization. AP Professional, Boston. 